\title{
A NEW STANDARD PULSAR MAGNETOSPHERE
}

\author{
IOANnis CONTOPOUlos $^{1}$, Constantinos Kalapotharakos ${ }^{2,3}$, and Demosthenes KaZanas ${ }^{3}$ \\ ${ }^{1}$ Research Center for Astronomy and Applied Mathematics, Academy of Athens, Athens 11527, Greece; icontop@academyofathens.gr \\ ${ }^{2}$ University of Maryland, College Park (UMDCP/CRESST), College Park, MD 20742, USA \\ ${ }^{3}$ Astrophysics Science Division, NASA/Goddard Space Flight Center, Greenbelt, MD 20771, USA \\ Received 2013 October 9; accepted 2013 November 25; published 2014 January 7
}

\begin{abstract}
In view of recent efforts to probe the physical conditions in the pulsar current sheet, we revisit the standard solution that describes the main elements of the ideal force-free pulsar magnetosphere. The simple physical requirement that the electric current contained in the current layer consists of the local electric charge moving outward at close to the speed of light yields a new solution for the pulsar magnetosphere everywhere that is ideal force-free except in the current layer. The main elements of the new solution are as follows: (1) the pulsar spindown rate of the aligned rotator is $23 \%$ larger than that of the orthogonal vacuum rotator; (2) only $60 \%$ of the magnetic flux that crosses the light cylinder opens up to infinity; (3) the electric current closes along the other 40\%, which gradually converges to the equator; (4) this transfers $40 \%$ of the total pulsar spindown energy flux in the equatorial current sheet, which is then dissipated in the acceleration of particles and in high-energy electromagnetic radiation; and (5) there is no separatrix current layer. Our solution is a minimum free-parameter solution in that the equatorial current layer is electrostatically supported against collapse and thus does not require a thermal particle population. In this respect, it is one more step toward the development of a new standard solution. We discuss the implications for intermittent pulsars and long-duration gamma-ray bursts. We conclude that the physical conditions in the equatorial current layer determine the global structure of the pulsar magnetosphere.
\end{abstract}

Key words: magnetic fields - pulsars: general

\section{INTRODUCTION}

The current theory of pulsars is founded on the assumption that the magnetosphere of a rotating magnetic neutron star will be filled with an electron-positron plasma that everywhere satisfies the force-free and ideal MHD conditions $\rho \mathbf{E}+\mathbf{J} \times \mathbf{B}=0$ and $\mathbf{E} \cdot \mathbf{B}=0$, respectively (Goldreich \& Julian 1969). Here, E and $\mathbf{B}$ are the magnetospheric electric and magnetic fields, and $\rho \equiv \nabla \cdot \mathbf{E}$ and $\mathbf{J}$ are the magnetospheric electric charge and current densities as measured by an inertial (nonrotating) observer. Based on this assumption, it has been shown that magnetic field lines cannot remain closed beyond the so-called light cylinder radius $R_{\mathrm{LC}} \equiv c / \Omega$ where $\Omega$ is the neutron star angular velocity, and that open magnetic field lines carry the electromagnetic energy responsible for the pulsar spindown. Contopoulos et al. (1999; hereafter CKF) first showed that the Goldreich-Julian requirements cannot be implemented everywhere in the magnetosphere. When one tries to obtain a solution that fills all space (and not one with regions of avoidance as in Lovelace et al. 2006), one finds that it necessarily involves an equatorial current sheet that provides closure for the large-scale electric current that flows from the star to infinity and back. In particular, the equatorial current sheet first discovered by CKF continues all the way to the stellar surface through two separatrix current sheets that meet at the so-called Y-point where the region of closed field lines ends on the light cylinder. This has now become the "standard" picture of the pulsar magnetosphere.

Unfortunately, the CKF solution (and all subsequent improvements and generalizations of it in ideal MHD) cannot say anything about how it is implemented microphysically. In other words, the solution for $\mathbf{E}$ and $\mathbf{B}$ requires a uniquely determined distribution of $\rho$ and $\mathbf{J}$, but there is no provision in the solution for where the required charges come from and how they are channeled around in the magnetosphere. This problem is most severe along the equatorial magnetospheric current sheet in the interior of which ideal MHD fails and dissipation of electromag- netic energy most probably takes place. Therefore, in order to understand how the ideal MHD global solution is implemented in practice, we need to focus on the physical conditions in the equatorial current sheet where the physical requirements are most severe.

Indeed, there has been strong recent interest in pulsar current sheet microphysics (Coroniti 1990; Lyubarsky \& Kirk 2001; Uzdensky et al. 2011; Uzdensky \& Spitkovsky 2014; Cerutti et al. 2013). All these studies focus on current sheet microphysics independent of the global structure of the magnetosphere. In a series of three articles (Contopoulos 2007a, 2007b, 2007c), we emphasized the strong coupling between the global magnetic field topology and reconnection in the equatorial current sheet. We showed that the nonideal MHD condition in the current sheet may dramatically modify the global electric current distribution and consequently the global magnetospheric solution as well. These results are confirmed in recent efforts to move away from ideal MHD with the introduction of magnetospheric dissipation through various ad hoc prescriptions for a finite plasma conductivity (Kalapotharakos et al. 2012; Li et al. 2012b).

In view of the recent efforts to determine the physical conditions in the pulsar current layer and motivated by our earlier 2007 work, we believe that there is a need to reconsider the main elements of the ideal force-free pulsar magnetosphere. We realized that by imposing a simple microphysical requirement in the equatorial current sheet we uniquely obtain a new pulsar magnetosphere solution that may be used as the new standard for calculating pulsar spindown, magnetospheric particle acceleration, and magnetospheric radiation emission.

\section{THE OLD STANDARD SOLUTION}

In the present work we consider only the axisymmetric case. We will work in a cylindrical system of coordinates $(R, \phi, z)$ centered on the pulsar and aligned with its rotation axis with 
$\phi$ an ignorable coordinate. We find it convenient to rescale our radial coordinate to $x \equiv R / R_{\mathrm{LC}}$. The magnetic axis may be aligned/counteraligned with the rotation axis, in which case we will call the pulsar an aligned/counteraligned rotator. We also introduce the function $\Psi=\Psi(x, z)$ as the magnetic flux contained inside cylindrical radius $x$ at position $(x, z)$. Let us begin with a short review of the main microphysical issues of the CKF solution where current sheets are treated as dissipationless tangential discontinuities.

1. The distribution of poloidal electric current

$$
I_{\mathrm{CKF}}(\Psi) \approx \begin{cases}\frac{\Omega \Psi}{4 \pi}\left(2-\frac{\Psi}{\Psi_{\text {open }}}\right) & \text { if } \Psi \leqslant \Psi_{\text {open }} \\ 0 & \text { if } \Psi>\Psi_{\text {open }}\end{cases}
$$

along open magnetic field lines is obtained as an eigenfunction of the problem by requiring that the solution crosses the light cylinder smoothly. Here, $\Psi_{\text {open }}=1.23 \Psi_{\text {dipole }}$, where $\Psi_{\text {dipole }} \equiv \pi r_{*}^{3} B_{*} / R_{\mathrm{LC}}, B_{*}$ is the polar value of the neutron star magnetic field, and $r_{*}$ is its radius. This contains an equatorial current sheet beyond the light cylinder $(x>1)$ with a surface charge density

$$
\begin{aligned}
\sigma(x, 0) & =2 E_{z}\left(x, 0^{+}\right) \\
& =2 x B_{R}\left(x, 0^{+}\right) .
\end{aligned}
$$

$\sigma$ is positive/negative, therefore, the current sheet consists mostly of outflowing positrons/electrons. Their origin may be related to pair formation at the Y-point. Note that

$$
\sigma\left(1^{+}, 0\right)=0
$$

right outside the magnetospheric Y-point on the light cylinder, since $B_{R}\left(1^{+}, 0\right)=0$ at that location. The fact that the equatorial current sheet does not only carry an electric current but is also charged has not been adequately emphasized in previous studies. As we will see below, this has some very important consequences for the global structure of the pulsar magnetosphere.

2. The equatorial current sheet closes onto the star through two current sheets along the separatrix between open and closed field lines. The separatrix current sheets are charged with a surface charge density

$$
\begin{gathered}
\sigma(x, z) \equiv E\left(x, z^{+}\right)-E\left(x, z^{-}\right) \\
=-x B_{p}\left(x, z^{+}\right) . \\
\left\{\left[1+\frac{\left[2 I\left(\Psi_{\text {open }}\right) /(R c)\right]^{2}}{x^{2}\left[1-x^{2}\right] B_{p}^{2}\left(x, z^{+}\right)}\right]^{1 / 2}-1\right\} .
\end{gathered}
$$

$\sigma$ is negative/positive, therefore, the separatrix current sheets consist mostly of inflowing electrons/positrons. ${ }^{4}$ Their origin too may be related to pair formation at the Y-point (Contopoulos 2009). Here, $B_{p}$ is the poloidal magnetic field.

\footnotetext{
4 For the history of this discovery, we must acknowledge that although CKF did indeed discover the magnetospheric current sheet associated with the poloidal magnetospheric current, it was the referee of their article, Professor Leo Mestel, who pointed out the presence of tangential discontinuities at the two separatrices.
}

3. Open field lines that cross the zero space charge (null) surface contain a distributed outflowing/inflowing electric current consisting mostly of inflowing electrons/positrons inside the null surface and outflowing positrons/electrons outside. Their origin may be related to pair cascades in the so-called outer gaps.

4. A small amount of open field lines cross the null surface twice and contain a distributed inflowing/outflowing electric current. The origin of its associated charge carriers is obscure.

5. Most open field lines around the pole do not cross the null surface. Those contain a distributed inflowing/outflowing electric current consisting mostly of outflowing electrons/ positrons. Their origin may be related to pair cascades on the pulsar polar cap or in the outer gaps.

The most important part of the magnetosphere is the equatorial current sheet that guarantees the global poloidal electric current closure. The current sheet experiences a pinching force due to its reversing surface magnetic field components $B_{\phi}\left(x, 0^{+}\right)=$ $-B_{\phi}\left(x, 0^{-}\right)$and $B_{R}\left(x, 0^{-}\right)=-B_{R}\left(x, 0^{-}\right)$. The new element that we want to emphasize here is that the current sheet is also charged because the electric field component perpendicular to it also reverses across it, i.e., $E_{z}\left(x, 0^{+}\right)=-E_{z}\left(x, 0^{-}\right)$. Therefore, the vertical electrostatic self-repulsion of the current sheet surface charge density $\sigma$ contributes to its vertical support against the magnetic pinching force. Note that, in contrast to the solar corona, there is no "guide field" in the pulsar reversing equatorial current sheet as some recent investigations assume (Cerutti et al. 2013).

\section{THE EQUATORIAL CURRENT SHEET}

Let us now study the small-scale structure of the current sheet and assume that it has a thickness $2 h \ll R$. In the current sheet interior defined as $-h \leqslant z \leqslant h$, in general

$$
\begin{gathered}
B_{R}(x, z)=\frac{z}{h} B_{R}(x, h), \\
B_{z}(x, z)=B_{z}(x, h), \\
B_{\phi}(x, z)=\frac{z}{h} B_{\phi}(x, h), \\
E_{R}(x, z)=E_{R}(x, h)=-x B_{z}(x, h), \\
E_{z}(x, z)=\frac{z}{h} E_{z}(x, h)=x B_{R}(x, z), \\
E_{\phi}(x, z)=0 .
\end{gathered}
$$

Note that $E_{R}(x, 0)=B_{z}(x, 0)=0$ in CKF. In general, $E_{R}(x, 0)=x B_{z}(x, 0)>B(x, 0)$. We then have

$$
\begin{gathered}
\mathbf{J}_{\mathrm{cs}}=-\frac{B_{\phi}(x, h) c}{h} \hat{\mathrm{R}}+\frac{B_{R}(x, h) c}{h} \hat{\phi}, \\
\sigma_{\mathrm{cs}}=2 E_{z}(x, h)=2 x B_{R}(x, h), \\
\rho_{\mathrm{cs}}=\frac{E_{z}(x, h)}{h}=\frac{x B_{R}(x, h)}{h} .
\end{gathered}
$$



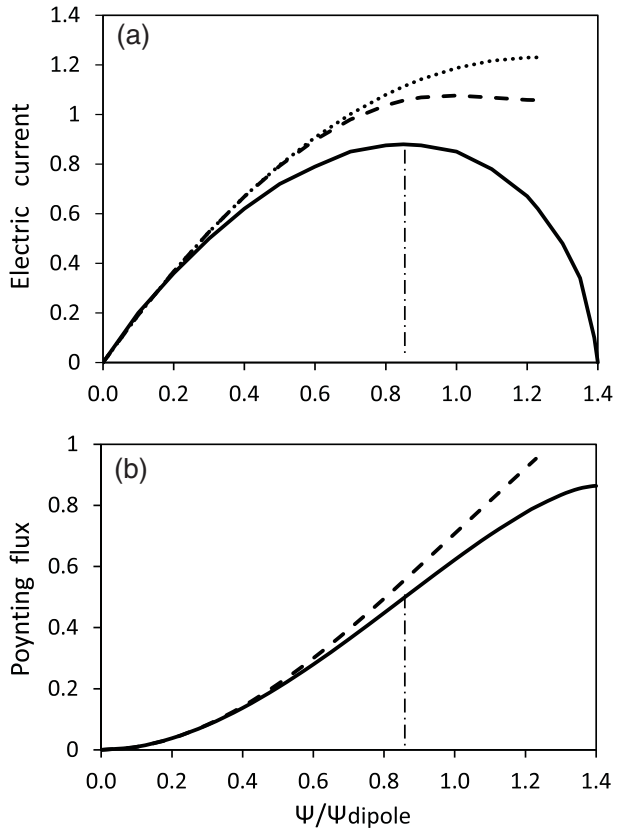

Figure 1. (a) Distribution of electric current $4 \pi I_{\text {New }} / c \Psi_{\text {dipole }}$ as a function of $\Psi / \Psi_{\text {dipole }}$ along "open" field lines. $\Psi_{\text {open }}=1.4 \Psi_{\text {dipole }} . I_{\text {New }}\left(\Psi_{\text {open }}\right)=0$. The solid/dashed/dotted lines indicate new/CKF/monopole solution, respectively. The dash-dotted line marks the value of $\Psi_{\max I}=0.85 \Psi_{\text {dipole }}=0.60 \Psi_{\text {open }}$ beyond which the return magnetospheric current flows. (b) Distribution of Poynting flux normalized to $E_{\mathrm{CKF}}$. The solid/dashed lines indicate new/CKF solution, respectively. The dash-dotted line marks the value of $\Psi_{\max }$ beyond which the magnetospheric Poynting flux gradually enters the current sheet.

The vertical force experienced by the current sheet plasma is equal to

$$
\begin{aligned}
F_{z}(x, z)_{\mathrm{cs}} & \equiv \rho E_{z}+\frac{J_{R} B_{\phi}-J_{\phi} B_{R}}{c} \\
& =\frac{\left[\left(x^{2}-1\right) B_{R}^{2}(x, h)-B_{\phi}^{2}(x, h)\right] z}{h^{2}} .
\end{aligned}
$$

In the old standard pulsar magnetosphere solutions, $z F_{z}$ is negative, i.e., the inward magnetic pinching force dominates. Most importantly though, at the points where the equatorial current sheet meets the light cylinder,

$$
F_{z}(1, z)=-\frac{B_{\phi}^{2}(1, h) z}{h^{2}} .
$$

In standard solutions, $B_{\phi}(1, h)=2 I\left(\Psi_{\text {open }}\right) /(R c) \neq 0$ and, therefore, $F_{z}(1, z) \neq 0$. An equivalent way to say that the magnetic pinching force in the current sheet dominates is to realize that the electric current in the current sheet in the old standard solution is spacelike, i.e., that

$$
\begin{gathered}
J_{\mathrm{cs}}=\frac{\left(B_{\phi}^{2}(x, h)+B_{R}^{2}(x, h)\right)^{1 / 2} c}{h} \\
>\frac{E_{z}(x, h) c}{h}=\rho_{\mathrm{cs}} c .
\end{gathered}
$$

This picture requires extra charge carriers of the opposite sign (electrons/positrons) in excess of the ones needed to account for $\rho_{\mathrm{cs}}$. It also requires a "hot" population of particles to support the current sheet against its own magnetic pinching.

A very simple and natural simplification to our problem is to assume that the electric current in the current sheet is everywhere null, or at least only weakly spacelike. In that case, Equation (13) yields $F_{z} \approx 0$. In other words, a null equatorial current sheet is electrostatically supported by its own charge and requires no additional "hot" population of particles. The above requirement amounts to a new equatorial boundary condition given implicitly through

$$
\frac{2 \Omega I(\Psi(x, h))}{c^{2}}=x\left(x^{2}-1\right)^{1 / 2} B_{R}(x, h)
$$

or equivalently

$$
\frac{\Omega I(\Psi(x, h))}{\pi c^{2}}=\left.\left(x^{2}-1\right)^{1 / 2} \frac{\partial \Psi(x, z)}{\partial z}\right|_{z=h}
$$

instead of the "standard" CKF equatorial condition $\Psi(x, h)=$ $\Psi(x, 0)=\Psi(1,0) \equiv \Psi_{\text {open }}$. Note that $2 I(\Psi(x, h)) /(R c)=$ $x B_{\phi}(x, h)$.

\section{THE NEW STANDARD SOLUTION}

As described in CKF and Timokhin (2006), we solve the pulsar equation numerically by iteratively relaxing both the distribution of $\Psi(x, z)$ everywhere in the half space $z>0$ (given the boundary conditions along the axis, infinity, and the equator), and the distribution of magnetospheric electric current $I(\Psi)$ that allows for a smooth and continuous crossing of the light cylinder. The main difference with $\mathrm{CKF}$ is the new equatorial boundary condition (Equation (17)) that is implemented during the iteration to evolve the equatorial distribution $\Psi(x, 0)$ for the next iteration step.

Our results are shown in Figures 1-3. In Figure 1(a) we show the distribution of poloidal electric current that we obtained along field lines that cross the light cylinder (we will still call them "open"). This can be fitted with the analytic expression

$$
\begin{gathered}
I_{\mathrm{New}}(\Psi) \approx \\
\begin{cases}\frac{1.07 \Omega \Psi}{4 \pi}\left(2-\frac{\Psi}{\Psi_{\mathrm{open}}}\right)\left(1-\frac{\Psi}{\Psi_{\mathrm{open}}}\right)^{0.4} & \text { if } \Psi<\Psi_{\text {open }} \\
0 & \text { if } \Psi \geqslant \Psi_{\text {open }}\end{cases}
\end{gathered}
$$

Here, $\Psi_{\text {open }}=1.4 \Psi_{\text {dipole }}$. For comparison, we also show the CKF current distribution and its corresponding monopole distribution. In Figure1(b) we show the distribution of Poynting flux in the pulsar magnetosphere, starting from the axis $(\Psi=0)$ up to the last open field line $\left(\Psi=\Psi_{\text {open }}\right)$. For comparison, we also show the CKF Poynting flux distribution.

The main elements of the new solution are as follows. (1) The pulsar spindown rate is equal to

$$
\dot{E}=0.82 \frac{B_{*}^{2} \Omega^{4} r_{*}^{6}}{4 c^{3}},
$$

i.e., $82 \%$ of the CKF spindown or $23 \%$ higher than that of the orthogonal vacuum rotator. (2) Sixty percent of the magnetic flux that crosses the light cylinder, $\Psi_{\max }$, carries the one part of the magnetospheric current that consists of electrons/positrons that outflow to infinity. (3) The electric current closes along the other $40 \%$ that consists of positrons/electrons that gradually converge to the equator. (4) This transfers $40 \%$ of the total pulsar spindown energy flux in the equatorial current sheet, which is then dissipated in the acceleration of particles and in high-energy electromagnetic radiation within a few times the light cylinder radius. ${ }^{5}$ (5) There is no separatrix current

\footnotetext{
5 Ninety percent of the dissipated energy is dissipated within six light cylinder radii.
} 


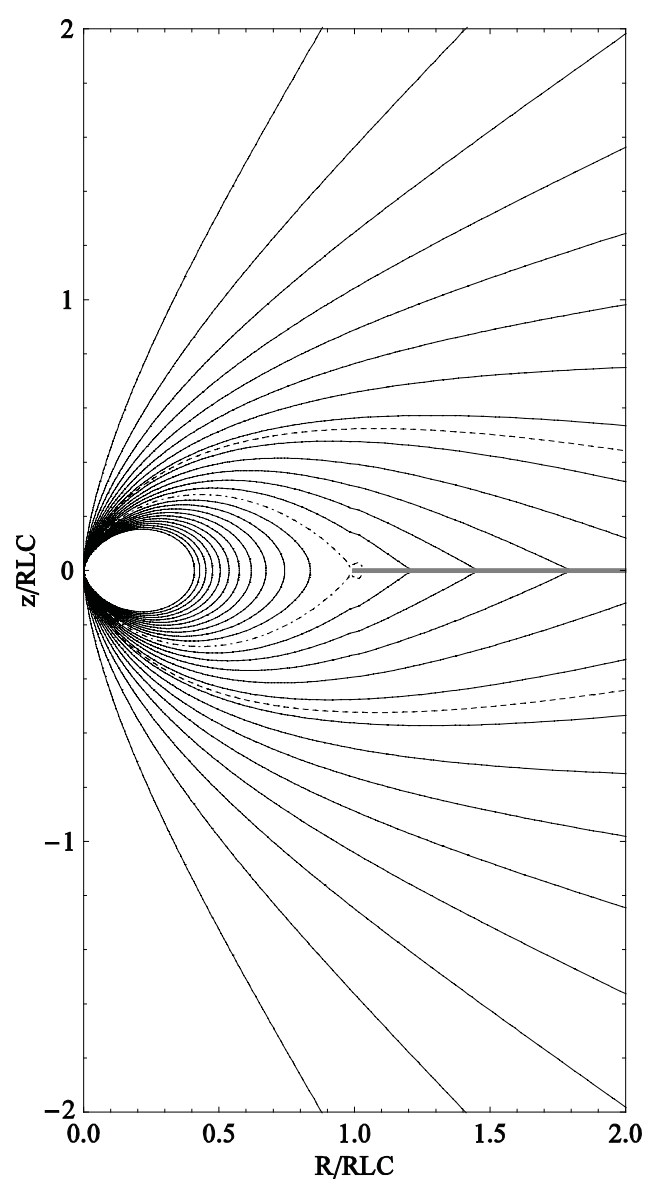

Figure 2. New structure of the pulsar magnetosphere with a null equatorial current sheet. Shown are magnetic flux $(\Psi)$ surfaces plotted in intervals of $0.1 \Psi_{\text {dipole. }}$ The dashed line indicates $\Psi_{\max I}=0.85 \Psi_{\text {dipole }}$ (corresponds to the boundary between outgoing and ingoing magnetospheric current, and asymptotically approaches the null line-i.e., the boundary between positive and negative space charge-and the equator as $R \rightarrow \infty)$. The dash-dotted line indicates $\Psi_{\text {open }}=1.4 \Psi_{\text {dipole }}$. The thick gray line indicates the equatorial current sheet.

sheet. (6) Pressure balance across the equatorial current layer is guaranteed by the electrostatic repulsion of the charge contained in it. Our solution is a minimum free-parameter solution in that the equatorial current sheet does not require a thermal particle population for vertical support against collapse.

The most important element of that solution is (5), namely the fact that

$$
I_{\text {New }}\left(\Psi_{\text {open }}\right)=0
$$

(Equation (17) for $x=1$ ), and $B_{\phi}(1, h)=0$. In other words, the electric current closes within the field lines that cross the light cylinder (we will still call them "open"), and thus there is no need for a current sheet along the separatrix between open and closed field lines. The equatorial current sheet is established to provide closure for the global pulsar electric circuit. As we said, only magnetic field lines that carry the return current consisting of outflowing positrons/electrons enter the current sheet and then outflow along the current sheet to infinity. The main magnetospheric current consists of electrons/positrons that outflow along the other $60 \%$ of magnetic field lines that originate closer to the polar cap axis. It is interesting that Equation (19) naturally resolves a complication in the CKF solution, namely that $\left.B_{p}(x, 0)\right|_{x \rightarrow 1^{-}}=2 I_{\mathrm{CKF}}\left(\Psi_{\text {open }}\right) \Omega /\left.c^{2}\left[x^{2}-1\right]^{1 / 2}\right|_{x \rightarrow 1^{-}} \rightarrow$ $\infty$ (Uzdensky 2003; Contopoulos 2009).

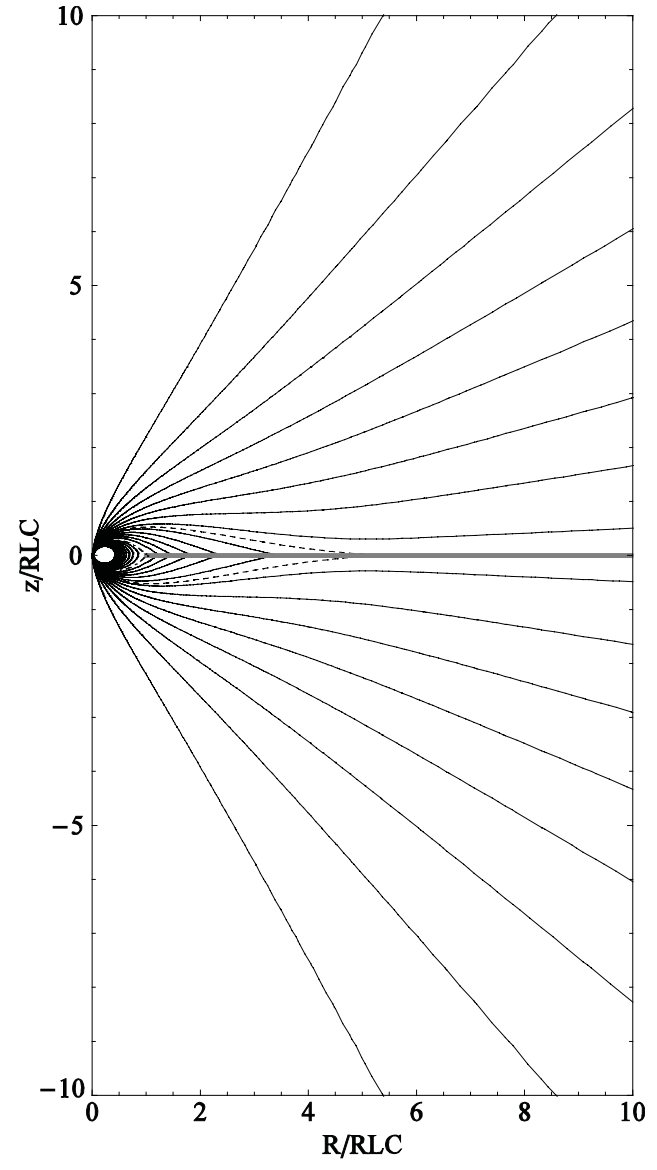

Figure 3. Same as Figure 2 but on a larger scale.

Note the qualitative similarity between the solution shown in Figures 2 and 3 and the solution obtained in Figure 5 of Contopoulos (2007c), which was based on analogous qualitative global arguments. In that solution, however, there was no provision for the ultimate opening up of field lines that carry the main magnetospheric current, and therefore the associated spindown rates obtained in that work are different.

\section{DISCUSSION}

We have presented a solution that we hope will become the new standard for calculating pulsar spindown, magnetospheric particle acceleration, and magnetospheric radiation emission. It is the first global solution that self-consistently takes care of the equatorial current sheet microphysical conditions in the limit of a null current. In that limit, the current sheet is self-supported electrostatically, and in that respect it is a "minimum parameter" solution, as it does not require an extra population of hot positrons/electrons. We must acknowledge that the electromagnetic (Poynting) energy that is deposited in the current sheet is dissipated in the acceleration of positrons/electrons by the $E_{R}=x B_{z}>B \neq 0$ electric field component in the current sheet (Equation (7)). Obviously, some fraction of those particles is expected to form a hot population that will partially contribute to the electrostatic support of the current sheet. In that respect, our present solution must be considered as one more step toward the development of a new standard solution. However, it is interesting that since particle acceleration proceeds beyond the light cylinder, any hot population will also form beyond the light cylinder. The absence of a hot 
population at the Y-point justifies one of the main results of the present work, namely the elimination of the separatrix current sheet.

The electrons and positrons that populate the pulsar magnetosphere may naturally be produced through pair production at the crossings of the zero space charge (null) surface, the so-called outer gaps. The electrons/positrons that move inward continue to flow through the polar cap and subsequently outflow in the main part of the polar cap, whereas the positrons/electrons that move outward reach the equatorial current sheet beyond the light cylinder (these are the ones that absorb the electromagnetic energy dissipated in the current sheet). Our minimum parameter solution is physically appealing in that it guarantees that pair production is not needed anywhere else in the magnetosphere.

Our solution addresses a fundamental issue that other ideal force-free solutions cannot address, namely, what is the fraction of the released electromagnetic energy that is deposited in the current sheet and dissipated in the acceleration of current sheet positrons/electrons. Our numerical integration yields $40 \%,{ }^{6}$ and it is expected that some significant fraction of it will be seen as high-energy pulsar radiation. We have thus found the main region of magnetospheric dissipation: the equatorial current sheet within a few light cylinder radii beyond the light cylinder.

Our results may also find application in the phenomenon of intermittent pulsars that are observed to spin down faster during their ON state than during their OFF state. If we associate the ON state with our present solution, the spindown rate of the ON state is only $82 \%$ of that of the CKF solution. We can naively generalize Equation (18) in three-dimensional in analogy to Spitkovsky (2006) as

$$
\dot{E}_{\mathrm{ON}}(\alpha) \sim 0.82 \frac{B_{*}^{2} \Omega^{4} r_{*}^{6}}{4 c^{3}}\left(1+\sin ^{2} \alpha\right),
$$

where $\alpha$ is the inclination angle between the magnetic pole and the rotation axis. If we also associate the OFF state with the Li et al. (2012a) solution with nearly vacuum conditions on the open field lines and nearly ideal force-free conditions on the closed field lines (where plasma remains trapped in the absence of pair production), then we obtain a ratio of respective spindown rates $\dot{E}_{\mathrm{ON}} / \dot{E}_{\mathrm{OFF}} 1-2.5$ for a likely range of pulsar inclination angles of $90^{\circ}-30^{\circ}$. This may naturally account for observed values between 1.5 and 2.5 .
Our solution may also find application in the study of longduration gamma-ray bursts (GRBs) where the central energy source is believed to be a newly formed maximally rotating stellar mass black hole that loses energy through electrodynamic processes very similar to the ones that take place in the pulsar magnetosphere (Blandford \& Znajek 1977; Komissarov \& Barkov 2009; Contopoulos et al. 2014). In particular, a maximally rotating $10 M_{\odot}$ black hole can lose up to about $10^{55}$ erg of rotational kinetic energy in the form of Poynting flux (Contopoulos et al. 2014). However, it is still not clear what fraction of that spindown energy will be radiated in the form of high-energy (X-ray, $\gamma$-ray) radiation. Our new standard solution yields a significant fraction of the spindown luminosity. If that result is confirmed, it will allow us to use long-duration GRBs as standard candles in Cosmology.

We conclude that the physical conditions in the equatorial current layer significantly modify the global structure of the pulsar magnetosphere.

This work was supported by the General Secretariat for Research and Technology of Greece and the European Social Fund in the framework of Action "Excellence."

\section{REFERENCES}

Blandford, R. D., \& Znajek, R. L. 1977, MNRAS, 179, 433

Cerutti, B., Uzdensky, D. A., \& Begelman, M. C. 2013, ApJ, 770, 147

Contopoulos, I. 2007a, A\&A, 466, 301

Contopoulos, I. 2007b, A\&A, 472, 219

Contopoulos, I. 2007c, A\&A, 475, 639

Contopoulos, I. 2009, MNRAS, 396, L6

Contopoulos, I., Kazanas, D., \& Fendt, C. 1999, ApJ, 511, 351 (CKF)

Contopoulos, I., Nathanail, A., \& Pugliese, D. 2014, ApJL, 780, L5

Coroniti, F. V. 1990, ApJ, 349, 538

Goldreich, P., \& Julian, W. H. 1969, ApJ, 157, 869

Kalapotharakos, C., Kazanas, D., Harding, A. K., \& Contopoulos, I. 2012, ApJ, 749,2

Komissarov, S. S., \& Barkov, M. V. 2009, MNRAS, 397, 1153

Li, J., Spitkovsky, A., \& Tchekhovskoy, A. 2012a, ApJ, 746, 24

Li, J., Spitkovsky, A., \& Tchekhovskoy, A. 2012b, ApJ, 746, 60

Lovelace, R. V. E., Turner, L., \& Romanova, M. M. 2006, ApJ, 652, 1494

Lyubarsky, Y., \& Kirk, J. G. 2001, ApJ, 547, 437

Spitkovsky, A. 2006, ApJ, 648, 51

Timokhin, A. N. 2006, MNRAS, 368, 1055

Uzdensky, D. A. 2003, ApJ, 598, 446

Uzdensky, D. A., Cerutti, B., \& Begelman, M. C. 2011, ApJ, 737, 40

Uzdensky, D. A., \& Spitkovsky, A. 2014, ApJ, 780, 3

\footnotetext{
6 That value will change when we incorporate partial thermal support in the
} current sheet beyond the light cylinder. 